\title{
La revolución hermenéutica como horizonte de integración cientifica: relación con el derecho y su historia
}

\author{
Recibido: 5 de agosto de 2020 • Aprobado: 9 de octubre de 2020 \\ https://doi.org/10.22395/ojum.v20n42a6
}

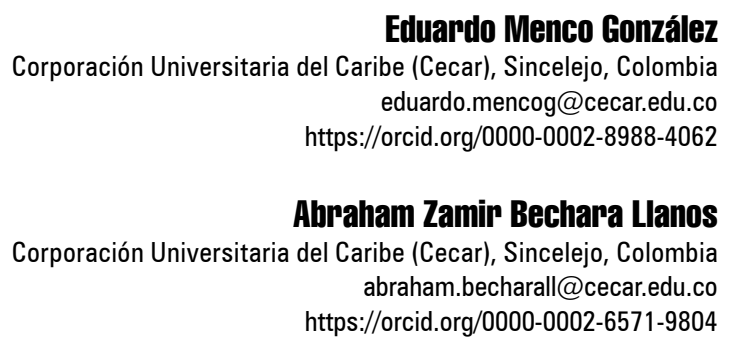

\section{RESUMEN}

El objetivo principal de este artículo es evidenciar los alcances de la revolución hermenéutica en el mundo contemporáneo como una nueva racionalidad de carácter interpretativo. Como elementos metodológicos para su concreción, tenemos la utilización del método histórico a partir del estudio de sus elementos tradicionales, el cual puso en entredicho la llamada racionalidad científica surgida a partir de los siglos XVII y XVIII. Como resultados de este trabajo presentamos las nuevas formas de comprensión del derecho y la ciencia jurídica, que están inmersas en la teoría de los sistemas jurídicos y de los modelos de aplicación del derecho en tiempos de Estado constitucional en la apertura del concepto de derecho en el siglo XXI a nuevas realidades y contextos jurídicos. Se concluye que la racionalidad hermenéutica es, para el derecho y otras perspectivas del conocimiento, un horizonte de interdisciplinariedad válido y necesario.

Palabras clave: hermenéutica; racionalidad científica; derecho; interdisciplinariedad; ciencia jurídica. 


\title{
The Hermeneutic Revolution as a Scientific Integration Horizon: the Relationship between Law and its History
}

\begin{abstract}
The main objective of the article is to show the scope of the hermeneutical revolution in the contemporary world, as a new interpretative rationality. As a methodological element for its accomplishment, the study employed the historical method based on the study of its traditional elements, which called into question the so-called scientific rationality that emerged from the seventeenth and eighteenth centuries. As a result of this, the study presents the new forms of understanding law and legal science immersed in the theory of legal systems, and of the models of application of law in times of constitutional State, in the opening of the same concept of law in the XXI century, to new realities and legal contexts. The study concludes that hermeneutical rationality is a valid and necessary horizon of interdisciplinarity for law and other perspectives of knowledge.
\end{abstract}

Keywords: hermeneutics; scientific rationality; law; interdisciplinarity; legal science. 


\section{INTRODUCCIÓN}

El objetivo de este trabajo de investigación, como ejercicio académico, es exponer una serie de consideraciones, no obstante su amplitud, acerca de la perspectiva interdisciplinar de la hermenéutica en relación con el derecho y su componente histórico. Asimismo, se presentan los elementos que, desde la teoría de la interpretación en sentido general y la hermenéutica constitucional, construyen la noción contemporánea de sistema jurídico. El artículo se enmarca en el proyecto en curso denominado Derechos fundamentales y región Caribe: un modelo de regionalización jurídica, financiado por la Corporación Universitaria del Caribe (Cecar), adscrito al Grupo de Investigación Giscer de la Facultad de Derecho y Ciencias Políticas.

La idea fundamental pretende poner de manifiesto cómo la revolución hermenéutica del siglo XX, expresada tanto en la filosofía en general como en la filosofía del derecho, ha abierto nuevas posibilidades hasta ahora inéditas en el camino de interdisciplinariedad e integración de los saberes que a todos preocupa como un asunto de amplísima relevancia. Particularmente desde el ámbito del derecho, se tratará de poner de manifiesto la integración de este con saberes más fundamentales o más verticales, como la filosofía o la teología que intentan dar una visión global de la realidad (Lyotard,1995); pero también con otras ramas del conocimiento científico, lo que nos sitúa quizás en una integración de carácter más horizontal.

Esta integración o interdisciplinariedad, que no es una mera yuxtaposición de saberes, presupone una ampliación de la racionalidad moderna tal como se entiende; aunque cada vez es menor en muchos ámbitos del conocimiento, sobre todo aquella racionalidad científico-técnica o matemática. Pero en la medida en que se ha desarrollado dicha ampliación, se puede aceptar la existencia de un camino para llevar a cabo aquella integración de saberes.

El ejercicio estará dividido en tres partes básicamente. En la primera parte se pretende hacer una brevísima introducción, a manera de diagnóstico, de cuál es la situación actual de la universidad hasta donde sea posible. En segundo lugar, se realiza un breve recorrido histórico con el cual se pretenderá mostrar cómo la revolución epistemológica de los siglos XVII y XVIII, sobre todo a consecuencia de la revolución científica, se proyectó sobre el derecho y configuró un modo de pensarlo que ha perdurado hasta la primera mitad del siglo XX que todavía se mantiene en buena medida. Finalmente, se intenta poner de manifiesto algunos puntos que son clave o compartidos en el pensamiento hermenéutico contemporáneo. Por último, se plantean una serie de conclusiones que enfatizan en cómo estas aportaciones de la hermenéutica, con sus diferentes corrientes y variantes, pueden ayudar a integrar el derecho con otras ciencias. 


\section{LA SITUACIÓN ACTUAL DE LA UNIVERSIDAD (BREVE CONSIDERACIÓN)}

Constituye un lugar común, al hablar de la universidad contemporánea, reconocer su fragmentación del conocimiento; no es algo nuevo, aunque es algo que posiblemente sea cada vez más intenso. En un discurso pronunciado por el presidente de la Universidad de Chicago en 1940 (García, 2018) en la Universidad de Yale se escuchó decir que el joven norteamericano entiende de forma accidental la tradición de la cual es parte y en la cual debe vivir, porque sus fragmentos dispersos y separados se encuentran regados de un extremo a otro del campus universitario. Los graduados universitarios tienen mucha más información y mucha menor comprensión que en la época colonial.

Se puede decir que tradición y comprensión son las palabras clave en este breve párrafo (parafraseado) del presidente de la Universidad de Chicago hacia finales de la primera mitad del siglo inmediatamente anterior.

Desde hace décadas la universidad vive una situación de crisis de identidad (García, 2015); no solo crisis económica, política o institucional, sino crisis en el sentido de pérdida de su identidad que va unida, como es obvio, a la incapacidad de dar una visión global, interpretativa y compleja del mundo desde cada una de sus disciplinas. La pérdida de esta visión integrativa va ligada a la deformación de algunos conceptos que han desaparecido o que son todavía marginales en la cultura contemporánea, tales como tradición, autoridad, verdad e, incluso, el concepto de comprensión.

En el ámbito del derecho este proceso de fragmentación no solo se manifiesta en la incapacidad que muchos juristas tienen para dialogar con otros científicos y con otras disciplinas del conocimiento, sino también en la grande dificultad que los juristas tienen para dialogar entre ellos (Atienza, 2018). Esto se debe a que el derecho, como otras ramas del conocimiento, se va especializando cada vez más y cada vez es más difícil y complejo encontrar un cierto discurso unitario y cohesionado.

No obstante, se pueden evidenciar algunos signos de cambio que vienen de la perspectiva de las investigaciones en la hermenéutica. Esta disciplina se entiende como el estudio de las condiciones que hacen posible la comprensión y la explicación de textos y acciones (Gadamer, 1998). La hermenéutica supone una especie de superación del paradigma racionalista, en la manera positivista de entender la razón y la apertura de esta a otros ámbitos o terrenos que quedaban cerrados al conocimiento hasta ese momento.

Para entender esta transformación que se ha operado desde la segunda guerra mundial, sobre todo a partir de los años sesenta en la filosofía y también, por supuesto, en la filosofía del derecho, parece importante y necesario hacer un breve recorrido histórico, volver a los orígenes y al cambio que se dio en su momento en los siglos XVII y XVIII con la revolución científica. 


\section{BREVE RECORRIDO HISTÓRICO}

Desde el siglo XII hasta la actualidad, la historia del derecho se puede dividir en dos grandes períodos o dos grandes experiencias jurídicas (Garrido, 2017): una que va desde el siglo XII propiamente hasta los siglos XVII-XVIII aproximadamente, y la otra inicia con la Modernidad (siglos XVIII al XX).

A partir de los siglos XVII y XVIII, como consecuencia de esta revolución de las ciencias y del conocimiento, los juristas y la ciencia jurídica tienden a construir un derecho "moregeométrico", esto es, un derecho que se parece, en su manera de razonar y en su forma de argumentar, a las llamadas ciencias exactas y experimentales. En este contexto, los nuevos valores que aparecen son los de certeza jurídica, seguridad, método, exactitud y el de previsibilidad. El motivo no era solamente científico o de revolución del conocimiento, sino que también había motivos económicos y políticos. De hecho, en este contexto se dio el desarrollo de una nueva cultura burguesa y de un incipiente capitalismo que hace necesario un orden jurídico que fuera cada vez más previsible, ordenado, sistemático y, por qué no, matemático.

Como fuente principal del derecho aparece la ley. Se da un proceso en entronización de la ley por primera vez en la historia, pues hasta entonces no sucedía de esta manera. La ley se mostró, entonces, como la única fuente del derecho capaz de darle a dicha ciencia la racionalidad exacta y matemática que se buscaba. Se da, así, una especie de canon de cientificidad que, además, fue la única que tuvo la capacidad de responder a las nuevas exigencias democráticas: asuntos como la costumbre y la interpretación judicial eran difícilmente encajables en estos nuevos cánones.

Como paradigma se puede citar la obra de Cesare Beccaria (2002) quien, como jurista, fue el autor de un libro pequeño con el cual pretendió condensar en pocas páginas toda una nueva manera de reflexionar la ciencia jurídica. Su obra, de carácter pernal, se denominó De los delitos y de las penas, en la que sustentó la idea de un nuevo orden jurídico a partir de un modelo antropológico novedoso de un contrato social de individuos aislados, esto es, individuos independientes que hacen un pacto estableciendo un "nuevo estado de cosas" imaginario donde la única fuente del derecho sería la ley. Según el autor, esta ley debe ser aplicada por los jueces haciendo un silogismo perfecto que consiste en una premisa mayor, la ley; una premisa menor, el hecho o la actuación; y como consecuencia o conclusión el delito, si es culpable o no.

En el contexto anterior, se suponía que la actuación de los jueces se convertiría en el resultado de su buena o mala digestión: si han comido bien, dictarían una sentencia favorable y si les ha sentado mal la comida, condenarían al reo (Feoli, 2015). Es la frase que ha pasado quizás más a la historia como interpretación del "nuevo estado de cosas" donde obviamente el papel del juez ha cambiado sustancialmente en relación con la manera en que anteriormente asumía su compromiso. 
Como fundamento de este nuevo modelo jurídico, que inicialmente se constituyó teóricamente, aparece una nueva concepción de la persona y de la sociedad basada en la idea de un individuo libre que se relaciona con los otros mediante un contrato. De esta manera, aparece el concepto de la autonomía de la voluntad que aún permanece en el ámbito jurídico. Además, se desarrolla la idea de los derechos del individuo, pero entendido como aislado con total independencia y capacidad de autohacerse: la época del secularismo y la mentalidad laical asomaba con rigor.

En definitiva, se puede entender este movimiento del derecho, que fue positivonegativo en muchos aspectos, como una reacción frente a todo el orden anterior: un orden jurídico existente desde el siglo XII hasta el XVIII que se basaba, sin temor a equívocos y simplificando mucho, en la persona del juez y su interpretación. Por tanto, era un orden jurídico poco dado a la seguridad pues, en efecto, dependía mucho de cómo el juez interpretara el derecho y estaba basado en una sociedad en términos corporativos. Esto quiere decir que los derechos se entendían en función de la pertenencia a un estatus, una corporación, un estamento, etc.; era una herencia de aquella idea medieval de populo Dei.

Este cambio de imaginario político-jurídico se llevó a la práctica, es decir, se institucionalizó a partir de las revoluciones liberales que, en buena medida, fueron el instrumento para que estas ideas teóricas intentaran hacerse realidad. En algunos contextos, esto se hizo de una manera brusca, como es el caso de la revolución francesa que fue una excepción en Europa, pero en otros casos se a través de revoluciones mucho más pausadas y más lentas (Hobsbawm, 2009). A lo largo del siglo XIX se puede decir que el derecho y la ciencia jurídica siguen básicamente estos parámetros fundacionales, aunque con muchos cambios, críticas y transformaciones, como es de suponer (Fernández et al., 2016).

Hasta aquí, a partir de esta evolución, se hace necesario destacar tres características del derecho (del modo de pensar el derecho más bien) que implican un modo de pensar la racionalidad y que se mantienen, en un sentido u otro, durante todo el siglo XIX hasta la Primera y la Segunda Guerra Mundial.

La primera característica es una concepción positivista del derecho y de la ciencia jurídica. Es positivista porque el derecho se entiende fundamentalmente como ley, es decir, como un objeto externo al jurista. Así, lo propio del jurista sería manipular la ley como un botánico puede estudiar una planta o un veterinario un animal. El derecho es asumido como objeto externo a la persona.

La segunda característica es reconocer que la ciencia jurídica se identifica fundamentalmente con su método. Recordemos que el XIX es el gran siglo de la metodología jurídica que, para algunos como Dworkin (2006) y Kelsen (1995), llega a extremos realmente increíbles de perfección y de afinamiento en la manera de razonar por 
parte de los juristas. El derecho busca una objetividad y una exactitud los más parecida posible a las que se pueden obtener en las ciencias exactas o experimentales.

En tercer lugar, el derecho se va separando o disociando poco a poco del resto de ciencias y disciplinas. En este sentido, se concibe a sí mismo como una ciencia autosuficiente, la cual construye sus propios presupuestos de partida y que pretende ser perfecta en sí misma. Se consolida en esta época, entonces, la idea de sistema para referirse al derecho: el derecho como sistema (Espinar, 2017).

Siguiendo con el recorrido, llegamos a la llamada crisis de la modernidad y los conflictos planetarios. En esta crisis no solo esta manera de pensar el derecho sufre profundas transformaciones, sino la forma de pensar la sociedad, la economía, la política y el Estado. Tras la Segunda Guerra Mundial, la experiencia nazi y las experiencias totalitarias, emerge, sobre todo en Alemania (Carvajal, 2010), una conciencia clara (la conciencia por parte de la clase jurídica en general) de proteger, mediante el derecho, determinados contenidos materiales de justicia como un asunto que corresponde a la naturaleza misma de su condición de juristas: los juristas se dedican, en teoría, a favorecer la justicia y a hacer la justicia. Uno de estos elementos va a hacer precisamente la tesis de la conexidad necesaria entre el derecho y la moral, tomada como herramienta teórica por Alexy para construir su argumento de la corrección (Molinares y Bechara, 2017). En este contexto, el derecho en tiempos de Estado constitucional pretende corregir los problemas de indeterminación normativa en el escenario de interpretación del juez constitucional.

La segunda guerra mundial puso de manifiesto que la ciencia jurídica había fracasado en su objetivo fundamental, sobre todo porque Alemania había sido el país donde el derecho había alcanzado mayores niveles de desarrollo teórico. Posterior a la experiencia de guerra, aparece la conciencia compartida por parte de la clase política y de los juristas de salvaguardar algunos contenidos materiales de justicia, algo que era intocable (por decirlo de alguna forma) por la voluntad humana o, en definitiva, por la voluntad del legislador.

Posterior a ello, se repensó un derecho que debe responder a la recuperación de la vida moral (Hincapié y Escobar, 2019) de una moral social y crítica que represente rasgos distintivos de la legalidad contemporánea. Mucho de estos elementos y tópicos de ese nuevo derecho se establecen a partir la concepción del Estado constitucional de derecho y la aplicación de nuevos métodos de adjudicación de las normas (Carrillo y Bechara, 2019) como son: la máxima de proporcionalidad, el principio de razonabilidad, la interpretación judicial para los derechos fundamentales y una argumentación especial iusfundamental.

Surgen de esta manera los modernos tribunales constitucionales cuya finalidad es preservar, entre otras cosas, el derecho y los derechos como contenidos de justicia 
frente a cualquier ataque por parte del poder público, esto es, los derechos por encima de las leyes para resumir el asunto. Nace también, en el ámbito de la ciencia jurídica, un deseo de superar el positivismo jurídico y el estatalismo (Noguera, 2011), que eran las corrientes dominantes que habían conducido al "desastre" sin que esto signifique que fueran las causas directas e inmediatas, pero que se mostraron un poco inútiles a la hora de evitar lo sucedido. Finalmente, la entronización de los derechos o el deseo de protegerlos suponía volver a colocar al juez en el centro del ordenamiento jurídico, algo que había sido marginado en el contexto de la revolución francesa en tanto el juez representaba al antiguo régimen. Adquirió, entonces, un papel importante porque el juez será quien tendrá la responsabilidad de salvaguardar los derechos. Este contexto se desarrolla en el sistema jurídico colombiano gracias a la interpretación judicial de los derechos fundamentales (Bechara, 2017) como puesta en marcha de la construcción pretoriana del Estado constitucional.

En este nuevo contexto histórico se produce, tanto en el ámbito filosófico como en el jurídico, la denominada revolución hermenéutica (Gadamer, 1996) que supone, entre otras cosas, una crítica de fondo a la racionalidad científica que hemos analizado. Con esto no se ha querido decir que los avances en el derecho durante el siglo XIX fueran superfluos. Por el contrario, en muchos casos se consideran irrenunciables y la ciencia jurídica sigue trabajando sobre ellos, pero debe reconocerse que esta racionalidad científica y jurídica decimonónica parecía eran insuficientes. Así, esta revolución hermenéutica supuso en buena medida una apuesta por una superación de este tipo de "estrechez racional", al menos para el derecho.

\section{HORIZONTE INTERDISCIPLINAR DE LA HERMENÉUTICA}

Llegó el momento para mencionar nombres como Gadamer, Ricoeur, Dilthey, o en, el ámbito jurídico, autores como Gesell, Kaufmann, Hassemer y Dworkin por el lado anglosajón, quienes dinamizarán las nuevas reflexiones de la nueva revolución. Sus posturas, tanto a nivel de filosofía como en el campo jurídico, no son coincidentes. Sin embargo, se puede decir que hay algunos puntos en común en esta hermenéutica que apunta fundamentalmente a un nuevo paradigma de racionalidad que permite abrir el derecho, la ciencia jurídica y posiblemente también otras ciencias a un diálogo interdisciplinar.

El primer punto lo constituye la afirmación de la historicidad de todo acto de interpretación (Gadamer, 2007) o comprensión. Se entiende por comprensión, en términos generales, un proceso de apropiación de sentido. Todo acto de interpretación y de conocimiento jurídico se sitúa siempre en un determinado horizonte histórico (Aarnio, Laporta y Atienza, 2010). La historia no es algo ajeno al derecho ni de lo cual se pueda prescindir. La comprensión aparece, por tanto, mediada por la historia y por el lenguaje, que no es solo un objeto o una serie de categorías que se pueden utilizar 
por el jurista, sino que se entienden además como un vínculo capaz de establecer relaciones entre las personas, pero también entre estas y el mundo.

El segundo punto está referido al protagonismo que aquellos autores le atribuyen a las precomprensiones, prejuicios o visiones anteriores o heredadas del mundo que todos tenemos como factores que son necesarios en todo proceso de interpretación y de conocimiento. De esta manera, se supera la idea, muy extendida en el ámbito ilustrado, de una razón desprovista de supuestos que es capaz de autofundamentarse, esto es, una razón que opera ahistóricamente. Estos prejuicios deben asumirse como elementos que posibilitan la interpretación. En definitiva, si no se tuviera un saber o bagaje previo, no podría conocerse nada. Todo acto de comprensión se entiende como la consecuencia de una pregunta que el intérprete hace al texto y el diálogo que se establece. De esta forma la interpretación y la comprensión en general se entienden como un proyecto que comienza en el intérprete y que va al texto y vuelve. Dilthey (2000) nombra este proceso como una dimensión anticipativa y proyectiva del acto de comprensión, es decir, en todo acto de comprensión el intérprete hace una anticipación de sentido que después comprueba si es razonable o no.

En el caso del derecho, por ejemplo, cuando un juez tiene que dictar sentencia, piensa casi espontáneamente en una solución o al menos en una aproximación. El juez no va primero a la norma para aplicarla, sino que primero atisba la solución en el caso concreto y ve una solución precisamente porque pertenece a una tradición. Así, de una manera reflexiva, surge la solución al caso. Después volverá a los textos para ver si esa solución tiene un fundamento razonable desde el punto de vista jurídico a partir de la ley, la jurisprudencia, los principios del derecho, etc. En este sentido, desde las fuentes volverá al caso concreto para ajustar la situación, por lo que hay un ir y venir del texto al intérprete (circulo hermenéutico); un movimiento que no debería terminar nunca, pero que al final termina porque el juez debe dictar sentencia.

El tercer punto tiene que ver con la recuperación de la racionalidad práctica en el ámbito del derecho que pone en acción tanto un componente axiológico como otro lógico-argumentativo. Interpretar en el derecho, como en otras ciencias, es comprender y explicar, es lo que se denomina como "arco hermenéutico": uno comprende y explica, y cuanto más explica mejor comprende (Ricoeur, 2008). Por tanto, la comprensión/ interpretación no eximen del racionamiento y de la argumentación: son elementos fundamentales en todo proceso de interpretación. Este proceso hermenéutico, a su vez como praxis interpretativa, permite establecer conexiones axiológicas (Marques, 2018) en el uso del lenguaje jurídico frente a normas que, por su característica estructural, necesitan un proceso de concreción en la actividad judicial.

El cuarto y último asunto tiene que ver con el cuestionamiento de la neta separación entre sujeto y objeto, pues entre uno y otro se da una relación de pertenencia y distancia (Ricoeur, 2017). El sujeto no es absolutamente ajeno al objeto de conocimiento, pero 
tampoco se identifica con él. En el ámbito jurídico, lo anterior significa que el derecho no es una realidad externa al jurista que se pueda conocer de una manera objetiva y se pueda manipular indiscriminadamente, más bien se trata de que el jurista se sitúa en el derecho y recibe un sentido que viene de su tradición, formación y cultura para interpretar nuevos sentidos y obtener comprensiones (Dworkin, 2014).

En este punto viene bien el concepto de verdad de los griegos (aleteia), de quienes heredamos la idea de que el sentido de las cosas no lo constituye el dato como tal, sino el proceso de búsqueda para encontrarlo en las cosas que, posteriormente al camino emprendido, asumimos como ciertas: la apertura del hombre a la verdad es una apertura histórica que trasciende la idea de que la verdad es simple adecuación del objeto con el intelecto. La verdad es búsqueda, es identidad con el deseo para comprender. Quien conoce se sitúa en el plano de lo humano y hace que la verdad tenga un valor distinto; es una especie de solidaridad entre persona y verdad. La verdad para la decisión judicial como integridad y como espacio de construcción de una hermenéutica pospostivista, ya desde la visión de Dworkin (Dytz y Ramos, 2019), se reclama para el derecho una interpretación que vincule elementos políticos, sociológicos y de justicia. Estos elementos construyen nuevos modelos hermenéuticos (Bechara y Vides, 2019), la gran mayoría de origen romano-germánico. La trascendencia de esto se da en la contextualización normativa como forma de marco hermenéutico a partir de las problemáticas comunes a nuestros territorios, culturas y realidades.

En el campo jurídico, la aproximación hermenéutica o interpretativa que parte de la solidaridad originaria de persona-verdad en función del sentido, se puede enlazar con el derecho natural, entendido como fuente originaria de sentido de todo acto de interpretación jurídica. Esto se concibe así desde un planteamiento que supera, por un lado, el relativismo tardomoderno que evita hablar de cualquier cosa parecida al derecho natural, así como del otro extremo en el cual también se puede incurrir: una especie de dogmatismo-iusnaturalismo que asume el derecho natural como una especie de ordenamiento completo que está previo a la historia o es preexistente a la misma, y en este caso lo único que hay que hacer es aplicarlo a la realidad. La nueva racionalidad hermenéutica propenderá por un reconocimiento de la historia humana con sentido y en búsqueda de este.

\section{CONCLUSIONES}

El acento puesto por la hermenéutica moderna en los elementos antes mencionados - el carácter mediador de la historia del lenguaje en todo acto de interpretación, la centralidad de la interpretación en la misma comprensión jurídica, el protagonismo del sujeto que interpreta como persona que existe en el mundo y que tiene una relación originaria con la verdad - apunta a una reformulación de la ciencia jurídica y de sus presupuestos racionales para hacerla capaz de dialogar con otras disciplinas sin perder 
su naturaleza jurídica, sin convertirse en moral, política, economía o cualquier otra disciplina del saber.

Es menester resaltar aquellos elementos que hacen posible, en la tradición hermenéutica, el estudio de aquellas disciplinas o de aquellos saberes que trabajan por la producción de un conocimiento crítico de la tradición desde la cual el jurista opera. Hemos visto que la hermenéutica y particularmente la hermenéutica jurídica parten del concepto de tradición como el elemento indispensable que inicia el proceso interpretativo (Atienza, 2013). No se entiende la tradición como la pura historia, sino como algo que pertenece a la propia existencia humana como tal; no es solo datos del pasado ni algo cerrado, sino que se vive en el contexto de una determinada tradición.

Esta constatación de la importancia de la tradición no solo debe llevar a repensar el concepto propiamente, sino también a estudiar los elementos que la conforman y que no son solo de derecho, sino de otras disciplinas que aportan una visión más integral de la realidad, como la filosofía o la teología que se cuestionan por los asuntos últimos de la existencia humana. ¿Qué es el hombre, qué es el mal, qué la muerte, etc.? La tradición remite a estos saberes fundantes como remite al resto de saberes que contribuyen a la formación de una determinada imagen del hombre o a una determinada concepción de la persona humana. No es casualidad que sea precisamente en el país germano donde la ciencia jurídica, en concreto algunos constitucionalistas, se han centrado en la relación "derecho constitucional-imagen del hombre". Esto no quiere decir que se pueda pasar directamente de la antropología al derecho por aplicación, pero sí que existe una conexión natural.

Otro elemento clave es reconocer que la mediación lingüística de todo acto interpretativo debe llevar al derecho y a la ciencia jurídica a relacionarse más con quienes se dedican a la lingüística para asegurar actos de interpretación correctos desde el punto de vista teórico y metodológico.

En el plano propiamente de la ciencia jurídica, este giro hermenéutico se ha traducido también en el protagonismo que se reconoce a los principios en la interpretación del derecho. Este ya no se entiende como la aplicación de leyes, reglas o supuestos de hechos sin más, sino como la realización de una acción inspirada por unos principios que son garantía de la racionalidad práctica del mismo derecho. Es cierto que estos principios no son capaces de llevar por sí mismos todo un proceso de interpretación jurídica, pero son necesarios en los procedimientos de interpretación y de creación del derecho porque la interpretación de esta ciencia está presente en todos los momentos que hacen posible su realidad y naturaleza científica. La interpretación es propia de todo acto de conocimiento jurídico y está inspirada en los principios (Alexy, 1988), tanto así que contemporáneamente esta manera de pensar ya es una fuerte tendencia, pues desde ella se cree que existe una permeabilidad del derecho 
a otras disciplinas del conocimiento que son colindantes, por ejemplo, la ética o la filosofía en general.

La aproximación hermenéutica al derecho debe ayudar a integrar esta ciencia con otros saberes. La interdisciplinariedad que tanto se busca pretende superar (no eliminar) modos de ser, hacer, trabajar y pensar que históricamente poco contribuyen a racionalidades más necesarias hoy en día. La necesidad de integración es una realidad urgente. Se insiste en que la interdisciplinariedad no es fruto de una mera yuxtaposición de saberes ni mucho menos se exige el abandono de la propia disciplina (que es un riesgo ciertamente); implica más bien una profundización en el propio saber que lleva a conectar con otras disciplinas que también ahondan en los presupuestos de su comportamiento, de manera que, de una forma natural, el derecho no solo se pone en comunicación con la filosofía, sino también con otras ciencias. Esta interdisciplinariedad tampoco es un mero uso instrumental de otros saberes (que es necesario); se trata más bien de un diálogo respetuoso de saberes cargado de reconocimiento que supone sentido en cada uno de ellos desde la perspectiva de la comunicación y el compartir. Esto es lo que se espera del derecho y del resto de las disciplinas y saberes del conocimiento en función del desarrollo y el crecimiento de la sociedad.

\section{REFERENCIAS}

Aarnio, A., Atienza, M. y Laporta, F. (2010). Bases teóricas de la interpretación jurídica. Editorial Fundación Coloquio Jurídico Europeo.

Alexy, R. (1988). Sistema jurídico, Principios jurídicos y Razón práctica. IV Jornadas Internacionales de Lógica e Informática Jurídicas. San Sebastián, España.

Atienza, M. (2013). Curso de Argumentación. Editorial Trotta.

Atienza, M. (2018). Filosofía del derecho y transformación social. Editorial Trotta.

Beccaria, C. (2002). De los delitos y las penas. Editorial Losada.

Bechara, A. (2017). Jurisprudencia de principios e interpretación de la Constitución: el escenario de la Corte Constitucional colombiana. Justicia, 32, 15-37. https://doi.org/10.17081/just.23.31.2903

Bechara, A. y Vides, M. (2019). Tres modelos y un mismo objeto de interpretación de los derechos fundamentales: Dworkin, Alexy y Sieckmann. Justicia, 24(36), 196-213. https://doi.org/10.17081/ just.24.36.3765

Carvajal, P. (2010). La historia del derecho y la historiografía jurídica alemana de siglo XX. Revista de estudios nistórico-jurídicos, (32), 453-468. http://dx.doi.org/10.4067/S07 16-54552010000100017

Carrillo, Y. y Bechara, A. (2019). Juez discrecional y garantismo: Facultades de disposición del litigio en el código general del proceso. Jurídicas CUC, 15(1), 229-262. http://dx.doi.org/10.17981/ juridcuc.15.1.2019.09

Dilthey, W. (2000). Dos escritos sobre hermenéutica: el surgimiento de la hermenéutica y los esbozos para una crítica de la razón histórica. Ediciones Istmo. 
Dytz, J. y Ramos, N. G. (2019). Coherencia y responsabilidad política de la decisión en Dworkin. Opinión Jurídica, 18 (36), 87-107. https://doi.org/10.22395/ojum.v18n36a4

Dworkin, R. (2006). La democracia posible: principios para un nuevo debate político. Paidós, Ibérica S.A.

Dworkin, R. (2014). Filosofía del derecho. Fondo de Cultura Económica.

Espinar, J. M. (2017). El derecho como sistema (Introducción a los estudios jurídicos). Editorial IGS XXI.

Feoli, M. (2015). El nuevo protagonismo de los jueces: una propuesta para el análisis del activismo judicial. Revista de derecho de la Universidad Católica del Norte, 22(2), 173-198. http://dx.doi.org/10.4067/ S0718-97532015000200006

Fernández, M., Rodríguez, V., Torres, J. y Vallejo, G. (2016). Fundamentos contemporáneos del derecho público: transformaciones necesarias. Universidad Católica de Colombia. https://repository.ucatolica.edu.co/ bitstream/10983/14296/4/Fundamentos-contempor\%C3\%A1 neos-del-derecho-p\%C3\%BAblico.pdf

Gadamer, H. (1996). Acotaciones hermenéuticas. Editorial Celesa.

Gadamer, H. (1998). El giro hermenéutico. Cátedra.

Gadamer, H. (2007). El problema de la conciencia histórica. Editorial Tecnos.

García, N. (2015) La universidad sufre una crisis de identidad. elEconomista.es https://www.eleconomista. es/campus/noticias/6484427/02/15/La-universidad-sufre-una-crisis-de-identidad.html

García, R. (2018, 21-24 de octubre). Desfragmentar la Universidad: el Core Curriculum como marco integrador de saberes. Documentos Core Curriculum, (1), 1-14. http://hdl.handle.net/10171/49512

Garrido, J. (2017). Ciencia del derecho en la escuela histórica y la jurisprudencia de conceptos. Derechos y libertades, (37), 207-232. https://e-archivo.uc3m.es/handle/10016/27156\#preview

Hincapié, A. y Escobar, B. (2020). Lenguaje y derecho. Una lectura teológico-política a partir de Walter Benjamin. Opinión Jurídica, 19(38), 137-157. https://doi.org/10.22395/ojum.v19n38a7

Hobsbawm, E. (2009). Historia del siglo XX. Editorial Crítica.

Kelsen, H. (1995). Teoría general del derecho y del estado. Traducción de Eduardo García Máynez. UNAM.

Lyotard, J. (1995). ¿Por qué filosofar? Ediciones Altaya.

Marques, E. (2018). Hermenéutica e interpretación constitucional sistemática axioteleológica. Opinión Jurídica, 16 (32), 169-187. https://doi.org/10.22395/ojum.v16n32a7

Molinares, V. y Bechara, A. (2017). Entre la interpretación y la norma: la tasa razonable de justificación constitucional. Revista de Derecho, 48, 163-188. http://dx.doi.org/10.14482/dere.48.10144

Noguera, A. (2011). La teoría del Estado y del Poder en Antonio Gramsci: claves para descifrar la dicotomía dominación-liberación. Nómadas: Critical Journal of Social and Juridical Sciences, 29(1). https://www.redalyc.org/pdf/181/181 18941013.pdf

Ricoeur, P. (2008). Hermenéutica y acción: de la hermenéutica del texto a la hermenéutica de la acción. Prometeo Libros.

Ricoeur, P. (2017). Hermenéutica: Escritos y conferencias 2. Editorial Trotta. 